\title{
Two-Sided Bounds on the Free Energy from Local States in Monte Carlo Simulations
}

\author{
A. G. Schlijper ${ }^{1}$ and B. Smit ${ }^{2}$
}

Received December 7, 1988; final March 20, 1989

\begin{abstract}
We show that a precise assessment of free energy estimates in Monte Carlo simulations of lattice models is possible by using cluster variation approximations in conjunction with the local states approximations proposed by Meirovitch. The local states method (LSM) utilizes entropy expressions which recently have been shown to correspond to a converging sequence of upper bounds on the thermodynamic limit entropy density (i.e., entropy per lattice site), whereas the cluster variation method (CVM) supplies formulas that in some cases have been proven to be, and in other cases are believed to be, lower bounds. We have investigated CVM-LSM combinations numerically in Monte Carlo simulations of the two-dimensional Ising model and the two-dimensional five-states ferromagnetic Potts model. Even in the critical region the combination of upper and lower bounds enables an accurate and reliable estimation of the free energy from data of a single run. CVM entropy approximations are therefore useful in Monte Carlo simulation studies and in establishing the reliability of results from local states methods.
\end{abstract}

KEY WORDS: Monte Carlo simulations; free energy calculation; lattice models.

\section{INTRODUCTION}

In Monte Carlo (MC) simulation studies, knowledge of the free energy is important for distinguishing stable states from metastable ones, by checking which has the lower free energy. This distinction frequently needs to be made in systems undergoing a first-order phase transition. Unfortunately, calculation of the free energy is difficult with the commonly used

\footnotetext{
'Koninklijke/Shell Exploratie en Produktie Laboratorium, 2280 AB Rijswijk, The Netherlands.

${ }^{2}$ Koninklijke/Shell Laboratorium Amsterdam, 1003 AA Amsterdam, The Netherlands.
} 
Metropolis algorithm. ${ }^{(1)}$ This algorithm provides easy access to thermal averages of local observables, but the entropy density (i.e., entropy per unit volume) of the system is, in general, not locally observable, and hence the free energy density is not easily obtained.

To obtain entropy and free energy estimates from MC simulations, a number of methods have been proposed, ${ }^{(2)}$ of which the thermodynamic integration method ${ }^{(3)}$ is probably the most popular. However, it requires many $\mathrm{MC}$ runs along a path from some thermodynamic reference state to the state of interest. Consequently, this method can be very costly. Moreover, it is not always easy to find a convenient reference state and reversible integration path.

Here, we consider a method for free energy calculation that is based on data from one run at the conditions of interest. It extends the local states method (LSM) of Meirovitch ${ }^{(4-6)}$ by including additional approximation formulas for the thermodynamic entropy density to enable a precise assessment of the results. The LSM is a method for lattice systems (although it might be extendable to continuum systems by using lattice approximations) which was developed on the basis of considerations from the stochastic models simulation method of Alexandrowicz. ${ }^{(7)}$ The method does not seem to have found much application, ${ }^{(8,9)}$ despite its obvious computational advantage and the fact that it has been shown to be very accurate for Ising and lattice gas models. We believe that this may be partly due to the manner of its original derivation, which does not provide an immediate understanding of its validity and range of applicability. This situation is altered by a reconsideration of Meirovitch's formulas (as well as other entropy approximation formulas based on the concept of local states) in the light of recent results on global Markov properties of thermodynamic equilibrium states of lattice systems. ${ }^{(10-13)}$ It was this observation that prompted the investigation reported here. We explain this point below.

In the LSM for, say, an Ising system, one selects a spin and a certain group of its neighbors, forming a cluster of spins for which the configuration probabilities are sampled. That is, during the $\mathrm{MC}$ run one keeps track of the number of occurrences of each of the possible spin configurations of this cluster. These numbers provide an estimate of the equilibrium probabilities of the cluster configurations; the entropy density $s$ (=entropy per lattice site or per spin) of the entire system is then estimated from these cluster configuration probabilities. We shall call the set of configuration probabilities for the cluster the local state on that cluster. (We note that this usage of the term "local state" differs from that of Meirovitch: Meirovitch uses the word "state" in the meaning of configuration.) Thus, the entropy density $s$ is approximated by a function, say $T_{A}(\rho)$ of the local 
state $\rho$ on the cluster $A$. The LSM prescribes the selection of $A$ and specifies the function $T_{A}$.

The basic ingredients of the LSM are also found in the cluster variation method (CVM). ${ }^{(14-17)}$ This is an extension of the mean-field and quasichemical treatments of lattice model thermodynamics. In the CVM the entropy density $s$ is again approximated by some function $T_{A}(\rho)$ of the local state $\rho$ on some cluster $A$; however, where in the LSM the probabilities that determine the local state are determined by $\mathrm{MC}$ sampling, in the CVM, which was developed as an analytical method, they are considered as free variables, to be determined by minimization of the associated free energy expression. In view of the conceptual similarities between the LSM and the CVM, it is interesting to observe that the methods typically select quite different clusters for $A$ and also propose different formulas for $T_{A}$.

This observation suggests that the possibilities of hybridization should be explored. Such hybridization (or combination) is even more interesting in the light of the theoretical results mentioned earlier: these results establish rigorously that the LSM approximation formulas correspond to a converging sequence of upper bounds on the entropy density $s$ of the thermodynamic (infinite) system in equilibrium, whereas the CVM formulas typically correspond to lower bounds. Combining the two in an MC procedure would thus enable a reliable assessment of the accuracy of the entropy estimate, and hence of the free energy estimate. (Another example of combining upper and lower entropy bounds, not related to the ones discussed here, may be found in ref. 18.)

In the scouting investigation reported here, we set out to compare a number of approximation formulas for the entropy density $s$ and to acquire some idea of the relative difference between the upper and the lower bounds. As test cases, we selected two two-dimensional models on the square lattice: the first was the spin-1/2 classical Ising model, and the second the five-state Potts model. The former model was chosen for the obvious reason that it has a complete analytic free energy expression, and the latter one because it is a simple model with a first-order transition and analytic results at the transition point.

We performed MC simulations of these models and sampled the local states to obtain the configuration probabilities that are the input for the entropy approximation formulas. We found that the upper and lower bounds on $s$ obtained by using clusters of only six points are typically less than $1 \%$ apart. For clusters of eight points, the difference is generally less than $0.2 \%$. Except for very large simulations, this intrinsic (i.e., method-related) inaccuracy is of the same order of magnitude as the statistical uncertainty. Moreover, it appears that even in the critical region 
an accurate determination of the free energy is possible. This is a consequence of the fluctuations in energy and entropy canceling one another.

This paper is organized as follows. In Section 2, we give a brief description of the CVM and the LSM approaches to entropy estimation from local states, in order to give the methods some intuitive appeal. We refer to the relevant literature for any rigorous justification. In Section 3, we present specific approximation formulas applicable to two-dimensional models with nearest-neighbor interactions. Section 4 describes the simulations and Section 5 discusses the results.

\section{LOCAL STATES METHODS FOR ENTROPY APPROXIMATION}

In this section we consider the two-dimensional square lattice to illustrate the two different approaches that come under the heading of local states methods, namely the LSM (note the capitalization, which is used to distinguish Meirovitch's specific implementation from the general conceptual approach) and the CVM. Both methods estimate the entropy density of the thermodynamic (infinite) system from the entropies of a few small parts of the lattice, which can be obtained directly from computer simulations.

We shall use the word "cluster" to designate any finite set of lattice sites. An occupation variable or spin $s_{i}$ is associated with a lattice site $i ; s_{i}$ can have any of $q \geqslant 2$ distinct values. A configuration $\omega_{X}$ on a cluster $X$ is a collection of spin values $\omega_{X}=\left\{s_{i}: i \in X\right\}$. The probability of finding the configuration $\omega_{X}$ on the cluster $X$, regardless of the configuration on the remainder of the lattice, is the cluster configuration probability $P_{X}\left(\omega_{X}\right)$. The thermodynamic state of the entire system is specified by the collection of all the functions $P_{X}(\cdot)$ for all clusters $X$. The set of configuration probabilities for some specific cluster $Y$ defines the local state for $Y$. We note again that in this terminology "state" refers to a probability distribution and is not the same as "configuration." Meirovitch uses the word "state" to mean "configuration"; since the phrase "local states method" also fits our definition of "state," we adopt it without change and, furthermore, apply it to approximations that do not use the original LSM formulas.

From the local state $P_{X}(\cdot)$ on the cluster $X$ we can obtain the cluster entropy $S[X]$ :

$$
S[X]=-k \sum_{\omega_{Y}} P_{X}\left(\omega_{X}\right) \log P_{X}\left(\omega_{X}\right)
$$

The thermodynamic entropy density (entropy per lattice site) $s$ is given by

$$
s=\lim _{X \rightarrow \mathbf{Z}^{2}} \frac{S[X]}{|X|}
$$


where $|X|$ is the number of sites in $X$. (This limit exists in the sense of van Hove if there is invariance under lattice translations. We only consider systems with translational invariance.)

The idea of local states methods is to estimate $s$ from a knowledge of local states $P_{X}(\cdot)$ for a limited number (often one) of clusters $X$. Both the CVM and the LSM improve greatly on the naive estimate $|X|^{-1} \cdot S[X]$, although in quite different ways. Both methods, however, utilize the fact that knowledge of $P_{X}(\cdot)$ not only enables the cluster entropy $S[X]$ to be calculated, but the cluster entropies $S[Y]$ for all subclusters $Y$ of $X$ can also be calculated: from the distribution $P_{X}(\cdot)$ the local state $P_{Y}(\cdot)$ may be obtained as a marginal distribution by summing over the redundant spin variables in $X \backslash Y$.

The CVM has been used extensively during the past decades as an analytical tool for calculating phase diagrams from molecular interactions (for some recent applications, see refs. 19-21) and its mathematical aspects are by now reasonably well understood. ${ }^{(10,11,22-24)}$ We refer to the literature $^{(14-17,24)}$ for various descriptions; here we shall present merely a plausibility argument to give the method some intuitive appeal. Consider an $n \times m$ rectangular cluster $R_{n m}$ by way of example. Then [cf. Eq. (2)]

$$
s_{n m}=(n m)^{-1} \cdot S\left[R_{n m}\right] \rightarrow s
$$

for $n, m \rightarrow \infty$. If $s_{n m}$ can be expanded as

$$
s_{n m}=s+\frac{a_{10}}{n}+\frac{a_{01}}{m}+\frac{a_{20}}{n^{2}}+\frac{a_{11}}{n m}+\frac{a_{02}}{m^{2}}+\cdots
$$

then the linear combination of cluster entropies

$$
\begin{aligned}
c_{n m} & =S\left[R_{n m}\right]-S\left[R_{n-1, m}\right]-S\left[R_{n, m-1}\right]+S\left[R_{n-1, m-1}\right] \\
& =s-\frac{a_{20}}{n(n-1)}-\frac{a_{02}}{m(m-1)}+\cdots
\end{aligned}
$$

also converges to $s$, but faster than the sequence $s_{n m}$. The expression $c_{n m}$ is the CVM approximation with $R_{n m}$ as the so-called basic cluster. It may be considered as being based on an extrapolation to the limit of the sequence $s_{n m}$. For other choices of the basic cluster, the CVM formalism produces similar linear combinations of cluster entropies as approximations to the limit entropy density $s$. We stress the fact that the reasoning presented above is not the one used in general practice, nor has it been used in theoretical analysis of the CVM. [The existence of an expansion as given by Eq. (4) has not been established, as far as we know.] The more 
general $^{(14-17)}$ and precise ${ }^{(10,11,22-25)}$ approaches are, unfortunately, not intuitively obvious and tend to rely on specialized mathematical techniques.

The mathematical foundation for Meirovitch's LSM formulas has only recently been established with complete rigor, but the physical picture behind the approach is easily explained. Let us consider the Ising model from the binary alloy point of view. One way of producing an equilibrium situation is to start with a small crystal and to let A and B atoms settle, one at a time, on the surface according to their preferences. These preferences are expressed by conditional probabilities: i.e., the probability of an A (B) atom settling on the crystal surface is conditioned by what is there already. In this setup the equilibrium situation is produced by a dynamic process, for which the equilibrium state is the stationary state. The natural entropy concept is now a conditional entropy, which measures the randomness associated with adding yet another site to the lattice, given the occupation of the existing part. ${ }^{(26)}$ In the thermodynamic limit of an infinitely large system, i.e., when the existing lattice is infinite in extent and thus fills an entire half of the space, this conditional entropy, denoted by $\tilde{s}$, is equal to the thermodynamic entropy density $s .{ }^{(13,22)}$ The practical usefulness of this approach derives from the fact that the thermodynamic equilibrium state has a certain Markov property: only the boundary layer of the existing lattice contributes to the conditioning; the depth of the boundary layer is determined by the range of the interaction. This Markov property has long been known for finite lattices, but it has only recently been proven for the infinite lattice in more than one dimension. ${ }^{(13)}$ As a result, $s$ can now be expressed as the conditional entropy of one site with respect to an infinite boundary layer. Meirovitch's original LSM formulas are an approximation to this expression in that conditioning takes place with respect to a finite (even small, of the order of ten sites) part of the boundary layer only. It may now also be intuitively clear why such an LSM formula is an upper bound on $s$ : in conditioning with respect to only a part of the boundary layer, fewer restrictions are placed on the freedom with which the site may be occupied, and this results in more randomness and a larger entropy.

\section{ENTROPY ESTIMATES}

In this section we list various entropy approximations from both the CVM and the LSM, which are applicable to two-dimensional models on the square lattice with nearest-neighbor interactions that are invariant under lattice translations and reflections. 


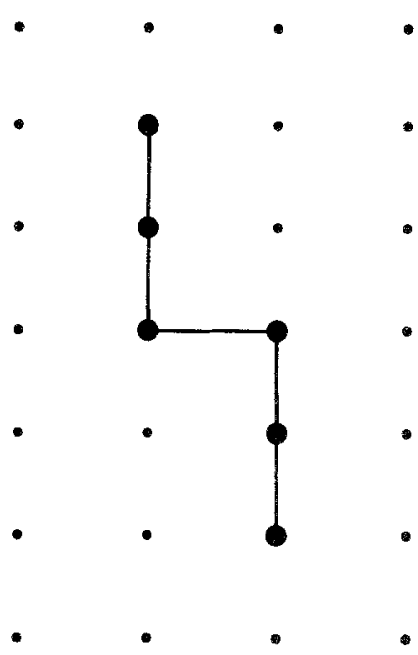

Fig. 1. The cluster $\mathrm{H}_{3}$.

For such models, the LSM considers Z-shaped clusters (Fig. 1) of $2 n$ sites, which we denote by $H_{n}$; the CVM in its traditional form uses rectangular clusters ${ }^{(27)} D_{n}$ (Fig. 2), although the cluster $H_{n}$ has been mentioned in this context, too. ${ }^{(11)}$ It is convenient to introduce some notation for subsets of $H_{n}$ and $D_{n}$ (see Fig. 3):

$$
\begin{aligned}
L_{n} & =\left\{z=\left(z_{1}, z_{2}\right) \in \mathbf{Z}^{2}: z_{1}=0,1 \leqslant z_{2} \leqslant n\right\} \\
R_{n} & =\left\{z=\left(z_{1}, z_{2}\right) \in \mathbf{Z}^{2}: z_{1}=1,-n \leqslant z_{2} \leqslant 0\right\} \\
O & =\{0\}=\{(0,0)\} \subset \mathbf{Z}^{2}
\end{aligned}
$$

so that

$$
H_{n}=L_{n-1} \cup O \cup R_{n-1}
$$

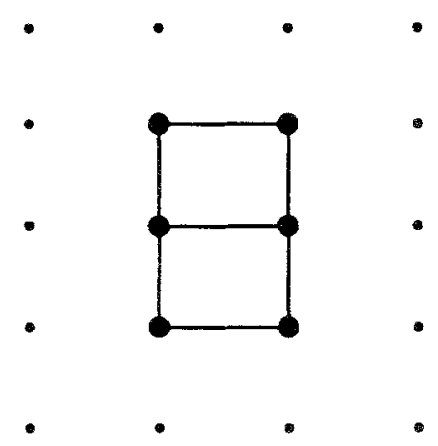

Fig. 2. The cluster $D_{3}$. 


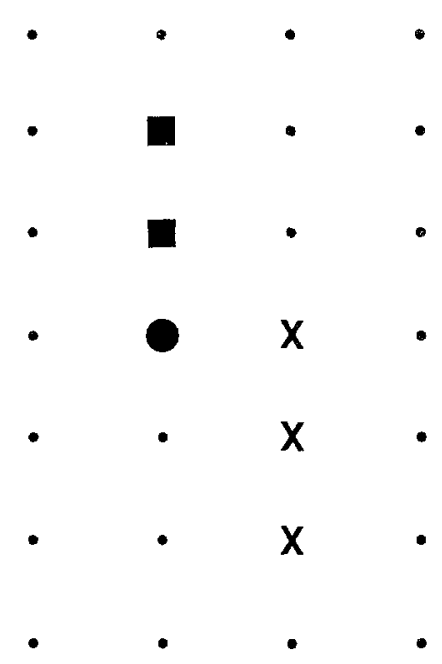

Fig. 3. Decomposition of the cluster $H_{3}$ into the subclusters $L_{2}, O$, and $R_{2}: \boldsymbol{\square}$, sites of $L_{2}$; - sites of $O$; $\times$, sites of $R_{2}$.

and $D_{n}$ may be written as

$$
D_{n}=L_{n} \cup \tau_{1} L_{n}
$$

where $\tau_{1}$ denotes translation over one lattice unit in the first coordinate direction. Note that any lattice site may be selected as $O$ because of translational invariance.

Meirovitch's entropy approximation, based on the cluster $H_{n}$, is

$$
d_{n}=S\left[H_{n}\right]-S\left[H_{n} \backslash O\right]
$$

which is a difference of two cluster entropies; this equals the conditional entropy of $O$ given the state on $L_{n-1} \cup R_{n-1}=H_{n} \backslash O$. In the limit of $n \rightarrow \infty$ the set $H_{n} \backslash O$ extends to cover the entire boundary layer, as described in the previous section. From the results of ref. 13, it follows that in thermodynamic equilibrium $d_{n}$ decreases monotonically to the entropy density $s$. Thus, the approximation $d_{n}$ is an upper bound on $s$, and the bound is closer if a larger cluster $H_{n}$ is used. The practical limitation is in the exponential increase, with increasing $n$, of the number of configurations on $H_{n}$ that must be sampled.

Application of the CVM formalism to the cluster $H_{n}$ results in the following entropy approximation:

$$
k_{n}=S\left[H_{n}\right]-S\left[L_{n-1} \cup R_{n-2}\right]-S\left[L_{n-1} \cup O\right]+S\left[L_{n-1}\right]
$$


Theorems A.I and A.II of ref. 11 yield the result that at equilibrium $k_{n} \leqslant s$ and also that $\lim k_{n}=s$; monotonicity of the convergence is not proven.

With $D_{n}$ as basic cluster, the CVM gives the following approximation to $s$ :

$$
c_{n}=S\left[D_{n}\right]-S\left[D_{n-1}\right]-S\left[L_{n}\right]+S\left[L_{n-1}\right]
$$

which again has the by now familiar look of something like a second-order difference [cf. Eq. (5)]. Again, it can be shown rigorously that for thermodynamic equilibrium states $c_{n} \leqslant s$ and that $c_{n}$ converges to $s$ with increasing $n$; here, also, monotonicity is not proven. ${ }^{(10,11)}$

An upper bound on $s$ with $D_{n}$ as the basic cluster is provided by the expression $^{(10)}$

$$
b_{n}=\frac{1}{n}\left\{S\left[D_{n}\right]-S\left[L_{n}\right]\right\}
$$

but, since experience has shown this bound to be very loose for all reasonable values of $n$, it is of no practical value and is not considered further.

The inequalities

$$
\begin{gathered}
s \leqslant d_{n} \\
k_{n} \leqslant s \\
c_{n} \leqslant s
\end{gathered}
$$

hold for the thermodynamic equilibrium state of the infinite system. In simulation practice, the values for $d_{n}$, etc., will be calculated from empirical configuration probabilities obtained from a finite sample of configurations on a finite lattice. This introduces a finite-size effect and a statistical (finite-sample) uncertainty, which may lead to small violations of the theoretical inequalities.

\section{MONTE CARLO SIMULATIONS}

We have tested the use of CVM entropy approximation formulas in conjunction with the LSM upper bounds in simulations of the Ising model and the five-states ferromagnetic Potts model on the square lattice. For the Ising model, exact results for free energy and entropy are available for all temperatures ${ }^{(28)}$; for the Potts model, reference data from an analytical calculation are available at the critical temperature. ${ }^{(29)}$

We present results of simulations on a $64 \times 64$ lattice with periodic 
Table I. Simulation Results for the Ising Model on the $64 \times 64$ Lattice

\begin{tabular}{|c|c|c|c|c|c|c|c|c|}
\hline \multirow[t]{3}{*}{$\begin{array}{c}\text { Temperature } \\
\qquad / T_{c}\end{array}$} & \multicolumn{2}{|c|}{$\begin{array}{l}\text { Energy density } \\
-u / J\end{array}$} & \multicolumn{4}{|c|}{$\begin{array}{l}\text { Entropy density } \\
s / k\end{array}$} & \multicolumn{2}{|c|}{$\begin{array}{c}\text { Free energy density } \\
-\beta f\end{array}$} \\
\hline & \multirow[t]{2}{*}{$\mathrm{MC}$} & \multirow[t]{2}{*}{ Exact } & \multicolumn{3}{|c|}{ MC } & \multirow[t]{2}{*}{ Exact } & \multirow{2}{*}{$\begin{array}{l}\text { MC } \\
\text { best interval }\end{array}$} & \multirow[t]{2}{*}{ Exact } \\
\hline & & & $k_{4}$ & $c_{4}$ & $d_{4}$ & & & \\
\hline 0.50 & 1.9924 & 1.9924 & 0.0076 & 0.0076 & 0.0076 & 0.0076 & {$[1.7637,1.7637]$} & 1.7637 \\
\hline 0.60 & 1.9733 & 1.9733 & 0.0227 & 0.0227 & 0.0227 & 0.0228 & {$[1.4721,1.4721]$} & 1.4721 \\
\hline 0.70 & 1.9313 & 1.9312 & 0.0511 & 0.0511 & 0.0511 & 0.0511 & {$[1.2669,1.2669]$} & 1.2669 \\
\hline 0.80 & 1.8524 & 1.8524 & 0.0971 & 0.0971 & 0.0971 & 0.0972 & {$[1.1176,1.1176]$} & 1.1176 \\
\hline 0.90 & 1.7133 & 1.7131 & 0.1689 & 0.1690 & 0.1690 & 0.1691 & {$[1.0079,1.0079]$} & 1.0079 \\
\hline 1.00 & 1.4231 & 1.4142 & 0.2989 & 0.3010 & 0.3038 & 0.3065 & {$[0.9281,0.9309]$} & 0.9297 \\
\hline 1.10 & 1.1100 & 1.1095 & 0.4349 & 0.4351 & 0.4357 & 0.4356 & {$[0.8798,0.8804]$} & 0.8801 \\
\hline 1.50 & 0.6858 & 0.6850 & 0.5848 & 0.5848 & 0.5848 & 0.5850 & {$[0.7862,0.7863]$} & 0.7862 \\
\hline 2.00 & 0.4803 & 0.4790 & 0.6379 & 0.6379 & 0.6379 & 0.6382 & {$[0.7438,0.7438]$} & 0.7438 \\
\hline 3.00 & 0.3062 & 0.3047 & 0.6701 & 0.6701 & 0.6701 & 0.6704 & {$[0.7151,0.7151]$} & 0.7151 \\
\hline 4.00 & 0.2261 & 0.2249 & 0.6805 & 0.6805 & 0.6805 & 0.6806 & {$[0.7054,0.7054]$} & 0.7054 \\
\hline
\end{tabular}

Table il. Simulation Results for the $q=5$ Potts Model on the $64 \times 64$ Lattice

\begin{tabular}{|c|c|c|c|c|c|}
\hline \multirow[t]{2}{*}{$\begin{array}{c}\text { Temperature } \\
T / T_{c}\end{array}$} & \multirow[t]{2}{*}{$\begin{array}{l}\text { Energy density } \\
-u / J\end{array}$} & \multicolumn{3}{|c|}{$\begin{array}{l}\text { Entropy density } \\
s / k\end{array}$} & \multirow{2}{*}{$\begin{array}{c}\text { Free energy density } \\
-\beta f \\
\text { best interval }\end{array}$} \\
\hline & & $k_{3}$ & $c_{3}$ & $d_{3}$ & \\
\hline 0.50 & 1.9986 & 0.0036 & 0.0036 & 0.0036 & {$[4.6978,4.6978]$} \\
\hline 0.60 & 1.9930 & 0.0154 & 0.0154 & 0.0154 & {$[3.9162,3.9162]$} \\
\hline 0.70 & 1.9768 & 0.0444 & 0.0444 & 0.0444 & {$[3.3607,3.3607]$} \\
\hline 0.80 & 1.9386 & 0.1037 & 0.1037 & 0.1037 & {$[2.9494,2.9495]$} \\
\hline 0.90 & 1.8542 & 0.2194 & 0.2194 & 0.2195 & {$[2.6388,2.6389]$} \\
\hline 0.95 & 1.7724 & 0.3230 & 0.3230 & 0.3231 & {$[2.5140,2.5141]$} \\
\hline $1.00^{a}$ & 1.5241 & 0.6165 & 0.6179 & 0.6219 & {$[2.4077,2.4117]$} \\
\hline 1.05 & 1.1180 & 1.0907 & 1.0911 & 1.0933 & {$[2.3415,2.3438]$} \\
\hline 1.10 & 1.0225 & 1.1961 & 1.1962 & 1.1971 & {$[2.2878,2.2887]$} \\
\hline 1.20 & 0.9093 & 1.3126 & 1.3126 & 1.3128 & {$[2.2024,2.2026]$} \\
\hline 1.30 & 0.8372 & 1.3805 & 1.3805 & 1.3806 & {$[2.1368,2.1369]$} \\
\hline 1.40 & 0.7858 & 1.4253 & 1.4253 & 1.4253 & {$[2.0845,2.0845]$} \\
\hline 1.50 & 0.7462 & 1.4574 & 1.4574 & 1.4575 & {$[2.0417,2.0417]$} \\
\hline
\end{tabular}

${ }^{a}$ Exact results: for $T=T_{c}^{-}: \quad-u / J=1.4737, \quad s / k=0.6792, \quad-\beta f=2.4098 ;$ for $T=T_{c}^{+}$: $-u / J=1.4208, s / k=0.7414,-\beta f=2.4098$. 
boundary conditions, performed with the conventional Metropolis algorithm. After an equilibration period of $1000 \mathrm{MC}$ trials per spin, a new lattice configuration was subjected to analysis at intervals of $3 \mathrm{MC}$ trials per spin. In the analysis the number of occurrences of each of the possible cluster configurations on the clusters $H_{n}$ and $D_{n}$ was counted; for the Ising model, we used $n=4$, which involved keeping track of two times $2^{8}=256$ cluster configurations; for the Potts model, we used $n=3$, involving two times $5^{6}=15,625$ cluster configurations. Each simulation run analyzed 10,000 lattice configurations to yield estimates of the cluster configuration probability distributions (local states) on the $H$ and $D$ clusters. The entropy estimates $d, k$, and $c$ [cf. Eqs. (12)-(14)] were calculated from these data. The estimates are presented in Tables I and II, together with the simulation results for the energy and reference data from the analytical solutions. The statistical uncertainty of the MC results for energy and entropy approximations was estimated, from a subdivision of the runs, to be $\lesssim 0.5 \%$ for $T \neq T_{c}$ and $\lesssim 1 \%$ for $T=T_{c}$. The free energy results are more accurate, however, which explains the number of decimals used in the presentation of the data. Note that the simulations at the critical temperatures were as long (or short) as the others. Using a $96 \times 96$ lattice did not produce significantly different results.

\section{DISCUSSION}

The data in Tables I and II confirm the previous observation ${ }^{(4-6)}$ that the LSM approximations $d_{n}$ are, for small values of $n$, very close to the exact entropy density. The approximations $k_{n}$ and $c_{n}$, based on the CVM, are shown to be equally accurate. In combination, they define a narrow interval that determines the entropy density $s$ with an accuracy that is of the same order of magnitude as the statistical accuracy of the MC sampling procedure. The smallness of this interval in itself signals the intrinsic accuracy of both the LSM and the CVM approximations; the exact results provide an opportunity for an independent verification, which would not usually be possible.

The differences between upper bounds, lower bounds, and exact entropy values hardly rise above the level of statistical noise, and therefore it is difficult to draw any firm conclusions from these test results beyond the statement that the intrinsic accuracy of these local states methods is impressive. The lower bound $c_{n}$ does seem to be slightly better than $k_{n}$, but this difference, too, is hardly significant. The advantage of using $k_{n}$ is that both $k_{n}$ and the upper bound $d_{n}$ are calculated from the same local state on the cluster $H_{n}$; to obtain $c_{n}$, the local state on the rectangular cluster $D_{n}$ must also be sampled; this requires additional computational effort. 
It is only at the critical temperature that there is an appreciable difference between the various entropy bounds and the exact value. Here also a significant finite-size effect is apparent: the exact entropy density for the infinite Ising model does not lie between the bounds obtained from the simulation on the $64 \times 64$ lattice-even if the statistical uncertainty is taken into account. On the other hand, the entropy density of the $64 \times 64$ Ising model is estimated ${ }^{(30)}$ to be 0.302 , which is well within the approximation interval.

As a consequence of the variational principle, which states that the free energy density is a minimum for a state of thermodynamic equilibrium, fluctuations in energy and entropy tend to cancel. We observe that the bounds $k, c$, and $d$ fluctuate in tandem and follow the entropy fluctuations. Hence, the free energy density can be estimated with considerable reliability even from a relatively short $\mathrm{MC}$ run: the free energy is more easily determined than its separate contributions, energy and entropy. This is clearly seen from the results at $T_{c}$.

We conclude that local states methods provide a very direct way of obtaining good free energy estimates. Data from only one simulation run under the conditions of interest are needed, which makes the technique computationally cheap and the implementation straightforward. The fact that the entropy is calculated from approximation formulas rather than with the help of an exact relation can no longer be considered a drawback: if the original LSM approximation of Meirovitch, which gives an upper bound on $s$, is combined with other approximations, derived in the CVM and yielding complementary lower bounds, the accuracy of the results can be assessed directly. The problem of dealing with statistical fluctuations is greatly reduced if one considers the free energy rather than considering the entropy and energy separately: the free energy can be obtained even at the critical temperature with a simulation of ordinary length.

The observation that formulas from the CVM can be useful in the context of Monte Carlo simulations is, we think, interesting in itself. There is a vast reservoir of entropy approximations for all kinds of situations available in the CVM, which could be explored for use in MC simulations. Such simulation results might in turn yield useful insights into the behavior of the CVM as an analytical tool.

A number of aspects of local states methods deserve further study. In the investigation reported here, we have found that good results can be obtained by using a cluster of six or eight lattice sites. For interactions with longer range and for three-dimensional models it is to be expected that clusters of more sites must be used to obtain equally good results. The memory requirements for sampling the local state on the cluster are then likely to become a limiting factor. This problem has already received some 
attention, in the $\operatorname{LSM}^{(6)}$ as well as in the CVM; possibly techniques that were developed in the CVM context ${ }^{(25)}$ can be used in the present context, too.

There is a theoretical question concerning some frequently used CVM approximations for three-dimensional lattices. While it is believed that they correspond to lower bounds on $s$, a proof has not yet been given; the techniques that have been used to establish the proofs in the two-dimensional case do not seem to admit an easy extension to three dimensions.

Finally, the influence of the finite size of the lattice used in the simulation needs more careful attention. The finite-size effect enters only through the sampled local state, which is the local state that corresponds to the equilibrium state of the finite lattice, whereas the theory that underlies the approximation formulas calls for the use of the local state belonging to the equilibrium state on a lattice of infinite size. In that case, the approximation formulas bound the thermodynamic limit entropy density $s$. The finite-size effect may lead to a situation where the actual calculated interval no longer contains $s$. The simulations at critical conditions provide examples. It would be of interest to understand the finite-size scaling behavior of the entropy bounds. Since they are not related in an obvious way to thermodynamic quantities of the finite system, it is not clear if existing scaling techniques can be applied.

\section{REFERENCES}

1. N. Metropolis, A. W. Rosenbluth, M. N. Rosenbluth, A. H. Teller, and E. Teller, J. Chem. Phys. 21:1087 (1953).

2. K. Binder, J. Comp. Phys. 59:1 (1985).

3. J. P. Hansen and L. Verlet, Phys. Rev. 184:151 (1969).

4. H. Meirovitch, Chem. Phys. Lett. $45: 389$ (1977).

5. H. Meirovitch, J. Phys. A: Math. Gen. 16:839 (1983).

6. H. Meirovitch, J. Stat. Phys. 30:681 (1983).

7. Z. Alexandrowicz, J. Stat. Phys. 5:19 (1972).

8. H. Meirovitch, Phys. Rev. B 30:2866 (1984).

9. H. Meirovitch, M. Vasquez, and H. A. Scheraga, Biopolymers 26:651 (1987).

10. A. G. Schlijper, J. Stat. Phys. 35:285 (1984).

11. A. G. Schlijper, J. Stat. Phys. 40:1 (1985).

12. R. Kuik, Commun. Math. Phys. 115:177 (1988).

13. S. Goldstein, R. Kuik, and A. G. Schlijper, Commun. Math. Phys., to appear.

14. R. Kikuchi, Phys. Rev. 81:988 (1951).

15. T. Morita, J. Phys. Soc. Jpn. 12:753, 1060 (1957).

16. G. W. Woodbury, Jr., J. Chem. Phys. 47:270 (1967).

17. D. M. Burley, in Phase Transitions and Critical Phenomena, Vol. 2, C. Domb and M. S. Green, eds. (Academic Press, New York, 1972), Chapter 9.

18. H. Meirovitch, Phys. Rev. A 32:3709 (1985). 
19. P. H. E. Meijer, R. Kikuchi, and P. Papon, Physica 109A:365 (1981).

20. G. M. Bell and G. L. Wilson, Physica 115A:85 (1982).

21. J. O. Indekeu, P. de Smedt, and R. Dekeyser, Phys. Rev. B 30:495 (1984).

22. A. G. Schlijper, Phys. Rev. B 27:6841 (1983).

23. A. G. Schlijper, J. Stat. Phys. 50:689 (1988).

24. S. J. Singer and J. D. Weeks, Phys. Rev. B 36:2228 (1987).

25. A. G. Schlijper and J. Westerhof, Phys. Rev. B 36:5458 (1987).

26. N. F. G. Martin and J. W. England, Mathematical Theory of Entropy (Addison-Wesley, Reading, Massachusetts, 1981).

27. R. Kikuchi and S. G. Brush, J. Chem. Phys. 47:195 (1967).

28. L. Onsager, Phys. Rev. 65:117 (1944).

29. F. Y. Wu, Rev. Mod. Phys. 54:235 (1982); R. J. Baxter, J. Phys. C 6:L445 (1973);

R. J. Baxter, H. N. V. Temperley, and S. E. Ashley, Proc. R. Soc. Lond. A 358:535 (1978).

30. H. Meirovitch and Z. Alexandrowicz, J. Stat. Phys. 16:121 (1977). 